\title{
Recurrent Immunodeficiency-Associated Burkitt Lymphoma Presenting as Severe Anterior Uveitis
}

\author{
Susan Huang ${ }^{a}$ Mark J. Suhrland ${ }^{b}$ David Gritz \\ Departments of a Ophthalmology and ${ }^{\mathrm{b}}$ Pathology, Albert Einstein College of Medicine, Montefiore Medical Center, \\ Bronx, N.Y., USA
}

\section{Key Words}

Burkitt lymphoma · Iritis · Anterior uveitis · Ocular lymphoma $\cdot$ Immunodeficiency

\begin{abstract}
Purpose: To report a case of recurrent immunodeficiencyassociated Burkitt lymphoma (BL) that initially presented as severe anterior uveitis. Methods: Case report. Results: To our knowledge, this is the first reported case of isolated anterior uveitis related to immunodeficiency-associated BL. A 34-year-old African American woman with a history of HIV and $B L$ in remission presented with unilateral anterior uveitis. Histopathologic study of an aqueous humor specimen was consistent for BL. Conclusion: The patient's initial presentation masqueraded as anterior uveitis, but her condition rapidly progressed with significant central nervous system involvement. Ocular involvement of immunodeficiency-associated BL is rare, with isolated anterior uveitis being even rarer. This must be kept in mind in the differential diagnosis for any patient with immunodeficiency, especially with a history of previous BL.

(c) 2015 S. Karger AG, Basel
\end{abstract}

\section{Introduction}

Burkitt lymphoma (BL) is a mature B-cell non-Hodgkin lymphoma initially described in 1958 as a mandibular malignancy in an African pediatric population [1]. It is associated with Epstein-Barr virus (EBV) infection [2-4]. There are three major variants of BL based upon their epidemiological distribution: endemic (African), sporadic (non-African), and immunodeficiency associated. BL is endemic in equatorial Africa, typically affecting the mandible, maxilla, and orbit [5]. The sporadic form occurs in nonendemic areas, including the United States, and it commonly affects the abdomen, viscera, bone marrow, and lymph nodes [6]. It rarely presents with ocular or orbital involvement [7]. The immunodeficiency-associated form presents similarly to the sporadic form, but has a more aggressive course affecting the central nervous system (CNS) [8].

We report a case of immunodeficiency-associated BL that presented as an isolated severe anterior uveitis. To the best of our knowledge, this presentation has not been described previously for BL in the literature.

\section{KARGER}

E-Mail karger@karger.com

www.karger.com/oop
(C) 2015 S. Karger AG, Basel

2296-4681/15/0022-0062\$39.50/0
Susan Huang, MD

Department of Ophthalmology

Montefiore Medical Center

3332 Rochambeau Avenue, 3rd floor, Room 306, Bronx, NY 10467 (USA)

E-Mail susan.huang16@gmail.com 
Fig. 1. Progression of pseudohypopyon of the right eye over time. a A 2.7-mm pseudohypopyon during the early course of the patient's presentation. b One month after the patient was lost to follow-up, the AC was dense with cells with layered inferior and temporal pseudohypopyon. c One week after slit-lamp photograph, the AC reaction increased. The inferionasal hymphema is iatrogenic from diagnostic paracentesis. d Two weeks after slit-lamp photograph with completion of one chemotherapy cycle, the AC reaction decreased with pseudohypopyon appearing as solid fibrin.
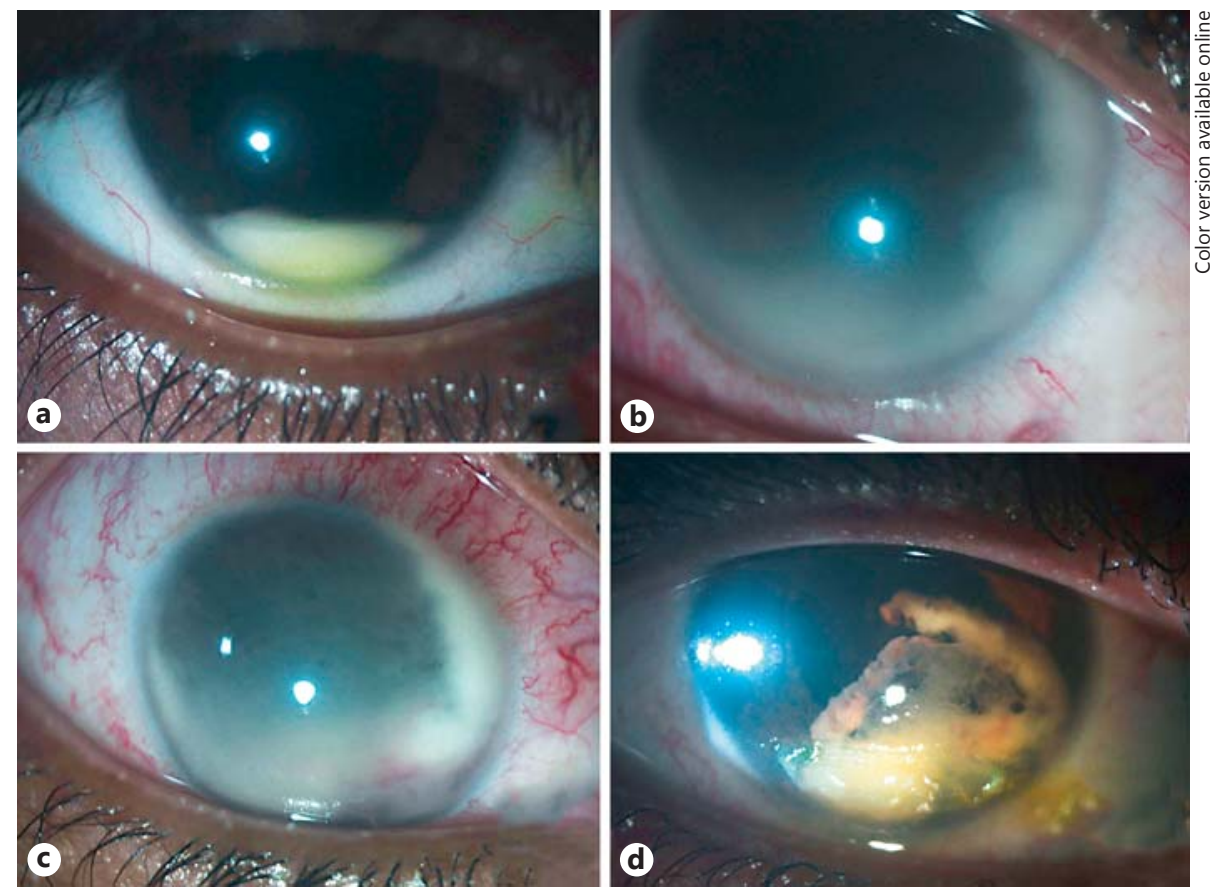

\section{Case Report}

A 34-year-old African American woman presented to the general eye clinic complaining of right eye redness, pain, photophobia, and blurred vision for 4 days. Her past medical history was significant for HIV on HAART therapy with a CD4 count of 194 and a history of BL status post-chemotherapy treatment 1.5 years previously with the hyper-CVAD regimen (cyclophosphamide, vincristine, doxorubicin, dexamethasone, methotrexate, and cytarabine). She was subsequently treated again 8 months prior to presentation with intrathecal methotrexate and cytarabine for suspicion of CNS lymphoma, but repeat cytology and flow cytometry were negative for malignancy after her treatment was completed.

The patient's initial ophthalmic evaluation revealed a best-corrected visual acuity of 20/30 in the right and left eye. Intraocular pressures were 26 and $13 \mathrm{~mm} \mathrm{Hg}$ in the right and left eye, respectively. She had normal extraocular movement and pupillary reaction without afferent pupillary defect. She had $1+$ conjunctival injection. The anterior chamber (AC) had 1-2+ cells, $2+$ flare with a 0.8 -mm hypopyon in the right eye. The anterior segment examination for the left eye was within normal limits. The dilated funduscopic examination was normal in both eyes. The presumptive diagnosis of right eye non-granulomatous anterior uveitis was given. The patient was started on prednisolone eye drops every $2 \mathrm{~h}$ while awake and cyclopentalate $1 \%$ twice daily in the right eye.

Subsequent close follow-ups over the next week revealed a rapidly increasing AC reaction to 3-4+ cells and hypopyon height to $1.8 \mathrm{~mm}$ despite increasing prednisolone eye drops to every hour in the right eye. Due to the worsening clinical presentation despite aggressive treatment, the differential diagnosis included BL. The patient was referred to the uveitis specialist to be seen within the following days.

Burkitt Lymphoma Presenting as Anterior Uveitis
The patient missed her appointment and returned to the clinic 2 weeks later. The hypopyon was $2.7 \mathrm{~mm}$, with the rest of the examination remaining stable (fig. 1a). AC diagnostic paracentesis was planned for the next visit to allow the coordination with the pathology department for the histopathology specimen. In the interim, prednisolone was increased to 3 times per hour in the right eye.

The patient was again lost to follow-up afterward. The ophthalmology consultant came into contact with the patient 5 weeks later when the patient was hospitalized for nausea and vomiting. Her visual acuity of the right eye deteriorated to hand motion. The intraocular pressure of her right eye was $36 \mathrm{~mm} \mathrm{Hg}$. Her extraocular movement of her right eye had full abduction but $25 \%$ movement of adduction, upgaze, and downgaze. New right upper eyelid ptosis was noted. The pupil could not be visualized due to the AC being fully dense with cells and layered hypopyon and fibrin temporally and inferiorly (fig. 1b). A B-scan of the retina showed the retina being flat without any evidence of vitritis. AC diagnostic paracentesis was performed at this time. Magnetic resonance imaging and magnetic resonance angiography were requested due to the patient's newly noted right oculomotor nerve palsy.

Histopathology of the aqueous humor was consistent with BL (fig. 2). The tumor cells showed positive immunoreactivity for the B-cell markers CD20 and CD10 and were negative for CD3 and BCL6. The Ki-67 labeling index was $80 \%$. Magnetic resonance imaging showed prominence and enhancement of bilateral multiple cranial nerves, consistent with the leptomeningeal spread of lymphoma.

Further systemic workup was performed for BL. Cytogenetic analysis did not show aneusomy or Myc rearrangement in 200 of 200 interphase nuclei. Flow cytometric analysis of the cerebrospinal fluid disclosed cells that were immunoreactive for CD10, CD19, CD20, and CD23, consistent with BL. It was negative for CD5, kappa, and lambda. Cerebrospinal fluid EBV was negative.

Ocul Oncol Pathol 2016;2:62-65 


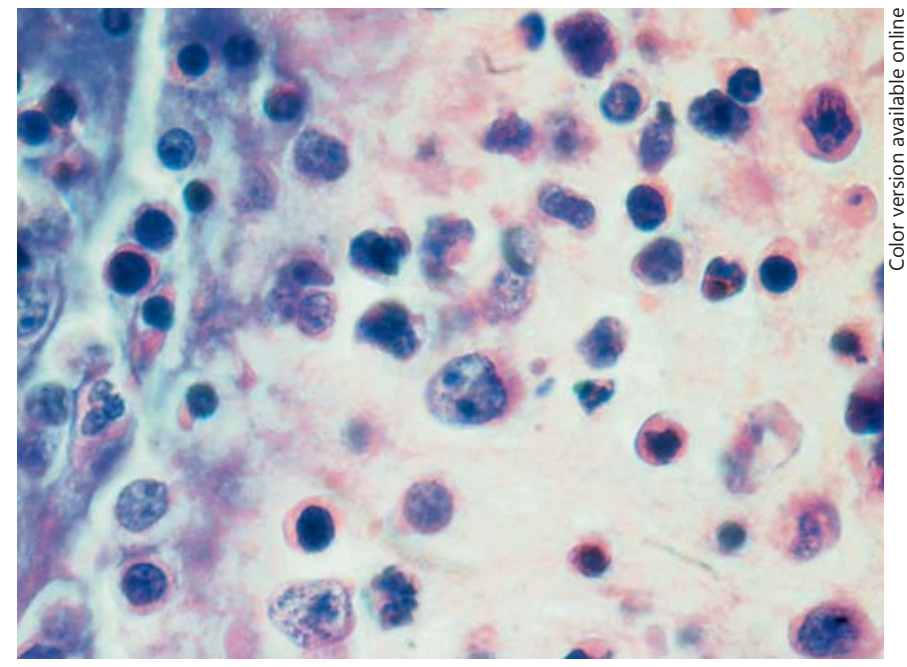

Fig. 2. Histopathology of an AC fluid specimen prepared as cell block showing tumor cells $12-14 \mu \mathrm{m}$ in size with a sparse to moderate amount of cytoplasm, multiple nucleoli, a mitotic figure, and numerous apoptotic nuclei. HE. $\times 1,200$.

Prior to starting chemotherapy while undergoing further workup, the extraocular movement became further restricted with full abduction but $0 \%$ movement in any of the other directions. The patient developed a new right facial droop. The AC reaction continued to worsen, with the dense pseudohypopyon and fibrin covering $80 \%$ of the AC height (fig. 1c).

The patient was started on chemotherapy with rituximab, methotrexate, and vincristine. After the first chemotherapy cycle, her vision improved to counting fingers at 3 feet. Her intraocular pressure decreased to $2 \mathrm{~mm} \mathrm{Hg}$. The AC reaction improved to $3+$ cells, and the pseudohypopyon, which now appeared as solid fibrin, covered $50 \%$ of the AC (fig. 1d).

Two weeks after completing the first chemotherapy cycle and starting palliative radiation therapy to the whole brain, vision was stable at counting fingers at 3 feet. The left eye was now starting to show a limitation with $15-20 \%$ abduction and adduction. The hypopyon and fibrin were resolved, with $3+$ cells in the AC.

Two weeks after the patient's last ophthalmology visit, the oncology team decided not to continue chemotherapy because the patient was not an appropriate candidate due to her poor physical condition and no improvement with the multiple cranial nerve palsies. She was started on home hospice afterwards.

\section{Discussion}

$\mathrm{BL}$ is a small, noncleaved B-cell non-Hodgkin lymphoma made up of monoclonal proliferation of B-lymphocytes [1]. It is highly aggressive with a doubling time of approximately $24 \mathrm{~h}$ [4]. The endemic form was found to be highly associated with EBV infection, with rates reported as high as $98 \%$. Sporadic BL has been associ- ated with EBV in $20 \%$ of the cases, and immunodeficiency-associated BL is related to EBV in approximately $40 \%$ of the cases $[7,9,10]$. It is thought that a defective regulation of EBV infection gives rise to uncontrolled B-cell differentiation in immunodeficiency-associated BL [9].

Immunodeficiency-associated BL typically has been described in HIV and organ transplant recipients [2, 3, 11 ]. It comprises $20-35 \%$ of the lymphomas in pediatric and adult HIV cases [9]. Immunodeficiency-associated BL shares similar clinical features as the sporadic type but has a higher incidence of CNS involvement $[12,13]$. The endemic form may commonly affect the orbit, but it is rare for the other two forms to have orbital or ocular involvement [7]. Even in spite of massive invasion of orbital tissues, it is uncommon for BL to have intraocular involvement [14].

Orbital and ocular involvements have been described with orbital invasion, cranial neuropathy, optic neuropathy, cavernous sinus infiltration with ophthalmoplegia, retinochoroidal and vitreous exudates, and optic nerve edema [7, 12, 14]. Baker et al. [7] reviewed sporadic BL cases and found the most common ophthalmic involvement without any orbital involvement to be cranial neuropathy in 7 out of 8 cases [15].

To the best of our knowledge, the initial clinical presentation of BL as anterior uveitis has not been previously reported. No previously published results were found through a literature search on PubMed. Although our patient had a prior diagnosis of $\mathrm{BL}$, she was thought to be in full remission for at least 8 months prior to her ocular presentation.

Awareness of potential ocular BL misdiagnosis needs to be recognized due to the rare occurrence of ocular involvement. With ocular or orbital involvement it is common to initially suspect infection or inflammation, with BL patients receiving antibiotics and/or steroids with no or transient improvement $[7,9,16]$. Due to our patient's progressive increase in pseudohypopyon over a 1-week period, BL was highly suspicious with its known rapid course [4]. Once BL is suspected rather than inflammatory anterior uveitis, diagnostic AC paracentesis should be performed for cytology.

Diagnosis of BL is confirmed by histopathology and immunophenotyping. The histologic pattern of small lymphoreticular cells combined with large, pale histiocytes has been referred to as 'starry-sky pattern,' which may be apparent on tissue biopsy [15]. The classic BL immunophenotypic profile is positive for the $\mathrm{B}$-cell markers CD10, CD20, CD21 (common to endemic BL), CD79a, 
BCL-6, and IgM, and negative for CD5, CD23, TdT, and BCL-20 [4, 9, 16, 17].

$\mathrm{BL}$ is frequently associated with the reciprocal translocations of the $c-M y c$ proto-oncogene from chromosome 8 with a different immunoglobulin heavy chain of chromosome $14 \mathrm{~b}$, which results in $c-M y c$ gene overexpression and thus in the monoclonal expansion of immature $\mathrm{B}$ cells [18-20]. $c-M y c$ rearrangements were detectable in $20-40 \%$ of the HIV-associated cases [20], which is consistent with our patient not having any detected chromosomal translocations.

Mortality from orbital and ocular BL is high, with rates of 54 and $88 \%$, respectively, of patients dying within the first year of presentation [7]. Poor prognostic factors include bone marrow involvement, initial CNS manifestation, and older age at diagnosis [16]. Our patient was placed in home hospice after 3 months from her initial ocular presentation.

In summary, we present the first known case of immunodeficiency-associated BL that masqueraded initially as anterior uveitis. BL should be considered in the differen- tial diagnosis of immunocompromised patients with anterior uveitis, especially in patients who were thought to be in remission from prior BL. Anterior uveitis may be the first clinical finding prior to any CNS symptoms, such as in our patient, who later on developed cranial nerve palsies. Although the uveitis-like condition may improve with chemotherapy, prognosis remains guarded due to the associated, rapidly progressive CNS involvement.

\section{Disclosure Statement}

The authors of this paper do not have any financial conflicts of interest.

\section{Statement of Ethics}

The patient was deceased at the time of preparation of this paper and thus was unable to provide her own consent. Her next of kin provided consent.

\section{References}

1 Burkitt D: A sarcoma involving the jaws in African children. Br J Surg 46:1958;218-223.

2 De-Thé G, Geser A, Day NE, et al: Epidemiological evidence for a causal relationship between Epstein-Barr virus and Burkitt's lymphoma from Ugandan prospective study. Nature 1978;274:756-761.

3 Wong M, Pagano JS, Schiller JT, et al: New associations of human papillomavirus, Simian virus 40, and Epstein-Barr virus with human cancer. J Natl Cancer Inst 2002;94:18321836.

4 Ziegler JL: Burkitt's lymphoma. N Eng J Med 1981;305:735-745.

5 Burkitt D, O’Connor GT: Malignant lymphoma in African children. I. A clinical syndrome. Cancer 1961;14:258-269.

6 Burkitt D: Burkitt's lymphoma outside the known endemic areas of Africa and New Guinea. Int J Cancer 1967;2:562-565.

7 Baker PS, Gold KG, Lane KA, Bilyk JR, Katowitz JA: Orbital Burkitt lymphoma in immunocompetent patients: a report of 3 cases and a review of the literature. Ophthal Plast Reconstr Surg 2009;25:464-468.
8 Giuliari GP, Sadaka A, Cortez MA, Paniagua A: Orbital Burkitt's lymphoma: an aggressive presentation. Case Rep Ophthalmol Med 2012;2012:354043.

9 Carmody J, Misra RP, Langford MP, Byrd WA, Ditta L, Vekovius B, Texada DE: Orbital sporadic Burkitt lymphoma in an adult diabetic African American female and a review of adult orbital cases. Clin Ophthalmol 2011; 5:509-515.

10 Shields JA, Shields CL, Scartozzi R: Survey of 1264 patients with orbital tumors and simulating lesions. The 2002 Montgomery Lecture, Part 1. Ophthalmology 2004;111:997-1008.

11 Gong JZ, Stenzel TT, Bennett ER, et al: Burkitt lymphoma arising in organ transplant recipients: a clinicopathologic study of five cases. Am J Surg Pathol 2003;27:818-827.

12 Levy J, Kratz A, Lifshitz T: Burkitt's lymphoma presenting as oculomotor palsy in an HIV-positive patient. Eur J Ophthalmol 2006; 16:186-189.

13 Nadal D, Caduff R, Frey E, et al: Non-Hodgkin's lymphoma in four children infected with the human immunodeficiency virus. Association with Epstein-Barr virus and treatment. Cancer 1994;73:224-230.

14 Payne T, Karp LA, Zimmerman LE: Intraocular involvement in Burkitt's lymphoma. Arch Ophthalmol 1971;85:295-298.
15 Trese MT, Krohel GB, Hepler RS, Naeim F: Burkitt's lymphoma with cranial nerve involvement. Arch Ophthalmol 1980;98:20152017.

16 Coupland SE, Krause L, Delecluse HJ, et al: Lymphoproliferative lesions of the ocular adnexa. Analysis of 112 cases. Ophthalmology 1998;105:1430-1441.

17 Odeoye AO, Durosinmi MA, Adeodu OO, et al: Ocular manifestations of Burkitt's lymphoma: experience in Ile-Ife South Western Nigeria. West Afr J Med 2007;26:48-52.

18 Gutierrez MI, Bhatia K, Barriga F, et al: Molecular epidemiology of Burkitt's lymphoma from South America: differences in breakpoint location and Epstein-Barr virus association from tumors in other world regions. Blood 1992;79:3261-3266.

19 Toujani S, Dessen P, Ithzar N, et al: High resolution genome-wide analysis of chromosomal alterations in Burkitt's lymphoma. PLoS One 2009;4:1-14.

20 Brady G, MacArthur GJ, Farrell PJ: EpsteinBarr virus and Burkitt lymphoma. J Clin Pathol 2007;60:1397-1402. 